\title{
EARLY ANTIINFLAMMATORY THERAPY ATTENUATES BRAIN DAMAGE AFTER SAH IN RATS
}

Abstract

Background: Early inflammatory processes may play an important role in the development of early brain injury (EBI) after subarachnoid hemorrhage $(\mathrm{SAH})$. Experimental studies suggest that anti-inflammatory and membranestabilizing drugs might have beneficial effects, although the underlying mechanisms are not fully understood. The aim of this study was to investigate the effect of early treatment with methylprednisolone and minocycline on cerebral perfusion and EBI after experimental SAH. Methods: Male Sprague-Dawley rats were subjected to $\mathrm{SAH}$ using the endovascular filament model. 30 minutes after $\mathrm{SAH}$, they were randomly assigned to receive an intravenous injection of methylprednisolone $(16 \mathrm{mg} / \mathrm{kg}$ body weight, $\mathrm{n}=10)$, minocycline $(45 \mathrm{mg} / \mathrm{kg}$ body weight, $n=10)$ or saline $(n=11)$. Mean arterial blood pressure (MABP), intracranial pressure (ICP) and local cerebral blood flow (LCBF) over both hemispheres were recorded continuously for three hours following SAH. Neurological assessment was performed after 24 hours. Hippocampal damage was analyzed by immunohistochemical staining (caspase 3). Results: Treatment with methylprednisolone or minocycline did not result in a significant improvement of MABP, ICP or LCBF. Animals of both treatment groups showed a non-significant trend to better neurological recovery compared to animals of the control group. Mortality was reduced and hippocampal damage significantly attenuated in both methylprednisolone and minocycline treated animals. Conclusion: The results of this study suggest that inflammatory processes may play an important role in the pathophysiology of $\mathrm{EBI}$ after $\mathrm{SAH}$. Early treatment with the anti-inflammatory drugs methylprednisolone or minocycline in the acute phase of SAH has the potential to reduce brain damage and exert a neuroprotective effect.

Keywords - subarachnoid hemorrhage $\cdot$ early brain injury $\cdot$ methylprednisolone $\cdot$ minocycline $\cdot$ neuroprotection

\section{Introduction}

The pathophysiology and treatment of early brain injury (EBI) have been more intensively focussed since drugs that successfully treated delayed cerebral vasospasm (DCV) failed to improve the neurological outcome of patients who suffered aneurysmal subarachnoid hemorrhage $(\mathrm{SAH}) \quad[1,2]$. The initial presentation, as documented by the Hunt/Hess and WFNS classifications, is still one of the most powerful predictors of long-term outcome. This further points out the importance of $\mathrm{EBI}[3,4]$. Furthermore, it cannot be excluded that early pathophysiological changes may significantly influence mechanisms of delayed neurological deterioration occurring later in the course of the disease $[5,6]$.

Apart from impaired cerebral perfusion, inflammatory pathways have been reported to contribute to $\mathrm{EBI}[7,8]$. It is assumed that the accumulation of hemoglobin in the subarachnoid space and contact with collagen fibers trigger an immunological reaction [7], resulting in the activation of immunomodulators and, finally, diapedesis of leukocytes and macrophages into the brain parenchyma. Degranulation of macrophages, in turn, may result in an amplification of immunological cascades as well as the formation of reactive oxygen species, damaging neurons and glial cells and cerebral blood vessels and thus causing necrotic and apoptotic cell death [9].

Because of its effect on the glucocorticoid receptor, methylprednisolone (MTP) interferes with the production and effect of inflammatory cytokines, chemokines and cell-adhesion molecules [10]. In addition, glucocorticoids could also target pathomechanisms which inhibit the prostaglandin synthesis early after SAH and cause early vasospasm [11]. Recently Gomis et al. reported the results of a randomized controlled clinical trial, that patients who were treated with MTP after SAH showed significantly better clinical recovery one year after SAH [12].
Georg Vadokas,

Stefan Koehler ${ }^{1}$

Judith Weiland'

Nadine Lilla',

Christian Stetter'

Thomas Westermaier ${ }^{1 *}$

'Department of Neurosurgery, University Hospital Würzburg, Josef-Schneider-Straße 11, 97080 Würzburg, Germany

2Department of Urology, Canisius Wilhelmina Hospital Nijmegen, Weg door Jonkerbos 100, 6532 SZ Nijmegen, Netherlands

Received 10 February 2019 accepted 18 March 2019
Minocycline $(\mathrm{MC})$ is a tetracycline antibiotic with the potential to inhibit inflammatory pathways occurring early after SAH. A neuroprotective potential has been observed in neurodegenerative diseases and traumatic brain injury [13, 14]. MC is a lipophilic drug and able to pass the blood brain barrier [15]. It exerts a number of anti-inflammatory mechanisms and may ameliorate the toxic effects of free subarachnoid hemoglobin, which is likely to be a trigger of inflammation after SAH $[14,16]$. It was the aim of this study to investigate the neuroprotective effects of MTP and MC on EBI after experimental $\mathrm{SAH}$.

\section{Material and Methods}

For the experiments, 35 male Sprague-Dawley rats (purchased from Charles River, Sulzfeld, Germany) with a body weight between $270 \mathrm{~g}$ and $370 \mathrm{~g}$ were used. All experiments were approved by the regional authorities and the district government of Bavaria, Germany. 


\section{Anesthesia and monitoring}

The rats were anesthetized with $4 \%$ Isoflurane (Isoflurane CP, CP-Pharma Handelsges. $\mathrm{mbH}$, Burgdorf, Germany), orally intubated and mechanically ventilated with an air/ oxygen/Isoflurane mixture. After induction of anesthesia, Isoflurane was reduced to $2 \%$ for surgical procedures and the following monitoring interval. Brain temperature was measured throughout the experiment using a temporalis muscle probe. With a heating lamp the temperature was kept at $37^{\circ} \mathrm{C}$ throughout the experiment. A polyethylene catheter (Portex ${ }^{\circledR}$ 0,58x 0,96mm, Smiths Medical, Kent, UK) was placed into the tail artery for continuous measurement of mean arterial blood pressure (MABP) and blood sampling. Arterial blood gases were measured 30 minutes and 5 minutes before induction of $\mathrm{SAH}$ and in hourly intervals thereafter.

\section{Intracranial pressure and local cerebral blood flow}

To measure the intracranial pressure (ICP), a burr hole was drilled over the right frontal cortex 3 $\mathrm{mm}$ lateral and $0.5 \mathrm{~mm}$ anterior of the bregma. The dura mater was opened diathermically. Two further burr holes were drilled $2 \mathrm{~mm}$ posterior and $5 \mathrm{~mm}$ lateral on each side of the bregma for the measurement of the local cerebral blood flow (LCBF) via laser-Doppler flowmetry (LDF). Care was taken not to injure the dura mater. After all burr holes were completed, the animals were placed in a supine position with their head fixed in a stereotactic frame with non-rupture ear bars. A Camino ICP-probe (Integra Neurosciences, Plainsboro, NJ, USA) was advanced $2 \mathrm{~mm}$ into the brain using a micromanipulator. Using two further micromanipulators, rectangularly bent laser-Doppler probes were positioned in the posterior burr holes, continuously measuring LCBF bilaterally in the area of the cerebral cortex supplied by the middle cerebral artery. The signal was recorded by a two-channel laserDoppler flowmeter (MBF3D, Moor Instruments, Axminster, England).

\section{Endovascular vessel perforation - induction of $\mathrm{SAH}$}

SAH was caused by applying the endovascular puncture technique, introduced by Bederson et al. [17]. After surgical exposure of the right cervical carotid bifurcation, temporary aneurysm clips were positioned on the common and internal carotid artery. A 3-0 Prolene $\odot$ filament (Ethicon, Inc., Somerville, NJ, USA) was inserted into the external carotid artery and fixed with a silk ligature. The aneurysm clips were removed and the filament was advanced into the internal carotid artery until ipsilateral LCBF decreased, indicating occlusion of the middle cerebral artery by the filament. The suture was then advanced an additional 2-3 $\mathrm{mm}$ for intracranial vessel perforation and then quickly withdrawn into the external carotid artery to allow instantaneous reperfusion of the internal carotid artery and development of SAH. $S A H$ was confirmed by a rapid bilateral decrease of LCBF and increase of ICP.

\section{Experimental groups and intervention}

Four animals died or were sacrificed before the randomization process due to irregularities in the induction of anesthesia $(n=2)$ or endotracheal intubation $(n=2)$. The remaining 31 animals were randomly assigned to one of the following groups:

1. Methylprednisolone (MTP) group $(n=10)$ intraperitoneal injection with $16 \mathrm{mg} / \mathrm{kg}$ body weight MTP (Urbason ${ }^{\circledast}$ solubile, Sanofi-Aventis Germany $\mathrm{GmbH}$, Frankfurt am Main), 30 minutes after $\mathrm{SAH}$

2. Minocycline (MC) group $(n=10)$ : intraperitoneal injection of $45 \mathrm{mg} / \mathrm{kg}$ body weight $\mathrm{MC}$ (Minocycline Hydrochloride, Sigma Aldrich, Darmstadt, Germany), 30 minutes after SAH.

3. Control group $(n=11)$ : intraperitoneal injection of $1 \mathrm{ml} / \mathrm{kg}$ body weight saline (B.Braun, Melsungen, Germany), 30 minutes after $\mathrm{SAH}$

At the end of the three-hour monitoring period all probes were removed and the wounds were closed with skin sutures. Anesthesia was withdrawn, analgesia was applied in form of $40 \mathrm{mg} / \mathrm{kg}$ body weight metamizole (Novaminsulfon ${ }^{\circledR}$-ratiopharm, Ratiopharm, Ulm, Germany) intraperitoneally and the rats placed into their home cages.

\section{Neurological assessment}

24 hours after SAH the animals underwent neurological testing including the assessment of hemiparesis and activity. The examinations were carried out by an examiner blinded to the animal's treatment arm. For neurological examination the animal was placed into an uncovered cage. After a ten-minute acclimatization interval, activity was evaluated by following a previously described observational protocol [18]. This protocol resembles a modification of the assessment of spontaneous activity reported by Garcia et al. in the framework of a largescale assessment protocol for evaluation of the neurological performance after experimental stroke.[19]. In brief, the animal was placed into a large uncovered cage enriched with several stimuli (paper towels, wood tunnel, cardboard box and food pellets) together with a non-operated animal. After 10 minutes of acclimatization, its activity was evaluated after repeated manipulation (tail-holding, lateral push, repeated displacement of the animal). Activity was graded following a 5-grade scale: 4) normal spontaneous activity, 3) slightly reduced spontaneous activity, 2) little or no spontaneous activity, but reaction on stimulus, 1) no activity on stimulus, 0) animal dead. The examiner was blinded to the animal's treatment arm. Thereafter, the presence of hemiparesis was tested by assessment of the limb movement and forepaw stretching.

\section{Quantification of subarachnoid} blood and histological assessment Following neurological assessment, the animals were again anesthetized with Isoflurane. Thereafter, an intraperitoneal injection of $1 \mathrm{ml}$ of sodium pentobarbital (Narcoren, Boehringer Ingelheim, Germany) was administered and the animals were transcardially perfused with $4 \%$ paraformaldehyde. The animals' brains were removed and extent of SAH was quantified under the operation microscope using the Sugawara grading scale [20]. A score ranging from 0 to 18 was given based of the amount of blood cloths in six segments of the basal cistern. Consequently SAH was classified as mild $(1-7)$, moderate $(8-12)$ or severe $(13-18)$.

After cryo-asservation the perfused brains were cut in $16 \mu \mathrm{m}$ coronary sections using a cryomicrotome (Leica CM3050s, Leica Mikrosystems Nussloch $\mathrm{GmbH}$, Heidelberg, 
Germany). Per animal two defined parts of the CA1 region of the hippocampus (bregma -3.72 and 4.92) were determined according to a stereotactic atlas of the brain [21]. The sections were stained with a Caspase-3 antibody (Cleaved Caspase-3 (Asp175) Antibody, 1:600, Cell Signaling Technology, Inc., Danvers, Massachusetts, USA) and co-stained with DAPI (4;6- Diamidino-2phenylindole, Sigma- Aldrich, St. Louis, Missouri, USA). The CA1 areas of the sections were bilaterally scanned for Caspase-3 positive cells per visual field under 40-fold magnification using a fluorescence microscope (Leica DMI 3000B, Leica Microsystems, Wetzlar, Germany). The number of damaged cells is depicted as a percentage of total visible cells in the visual field. The cell count was performed by an examiner blinded towards the animals' treatment arms.

\section{Statistical analysis}

Statistical analysis was performed using GraphPad Prism 4 (GraphPad Software, San Diego, California, USA). Data was tested for normal distribution using the D'Agostino and Pearson normality test. The measured end points of the experiment were compared using a one-way analysis of variance (ANOVA). When appropriate, Tukey's test for multiple comparisons was applied. The difference in mortality between the experimental groups was analyzed by a $X^{2}$-test. A p-value of < 0.05 was considered significant. Results are presented as mean \pm standard deviation (SD).

\section{Results}

\section{Physiological parameters}

Values of arterial blood gases are depicted in Table 1. There were no significant differences between the groups regarding arterial $\mathrm{pH}, \mathrm{pO} 2$ and $\mathrm{pCO}_{2}$ throughout the experiment.

\section{Mean arterial blood pressure, intracranial pressure and cerebral perfusion pressure}

Immediately before the induction of $\mathrm{SAH}$, MABP was $74 \pm 25 \mathrm{mmHg}, 68 \pm 9 \mathrm{mmHg}$ and $71 \pm 17 \mathrm{mmHg}$ in the control-, MTP-, and MC groups, respectively. After $\mathrm{SAH}$, a slight increase of MABP was recorded to reach maximum values of $81 \pm 20 \mathrm{mmHg}$ and $84 \pm 21 \mathrm{mmHg}$ after 5 and 60 minutes, respectively. In the treatment groups, MABP remained constant slightly below $70 \mathrm{mmHg}$ throughout the experiment with a minor increase at the end of the monitoring period to reach $74 \pm 26 \mathrm{mmHg}$ in the MTP group and $75 \pm 21 \mathrm{mmHg}$ in the MC group. Differences between the groups were not statistically significant (Figure 1a).
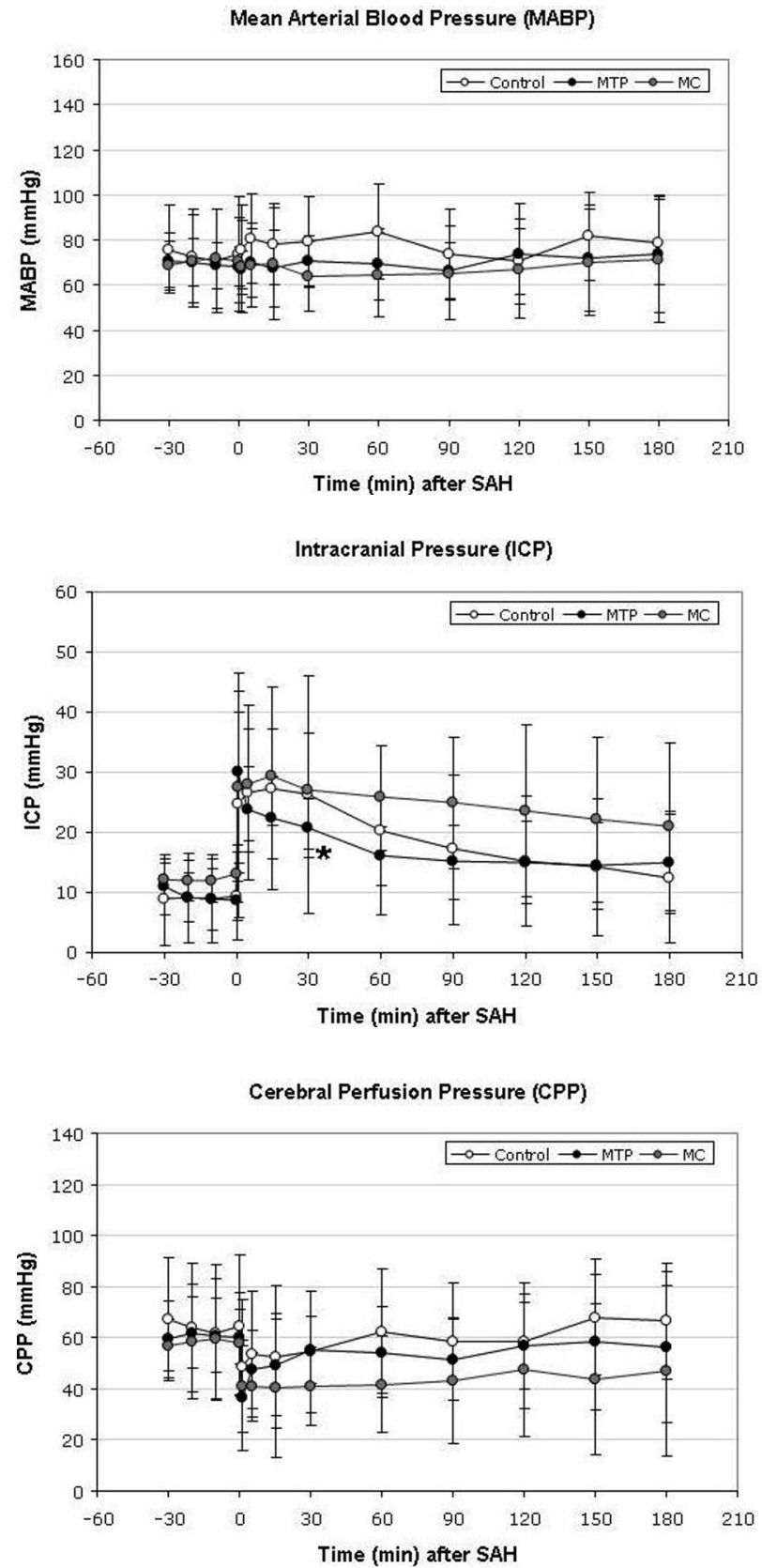

Figure 1a-c: Course of mean arterial blood pressure (MABP), intracranial pressure (ICP) and cerebral perfusion pressure (CPP) continuously measured from 30 minutes before until 180 minutes after induction of subarachnoid hemorrhage $(\mathrm{SAH})$. While there was no statistically significant between the groups regarding the course of MABP (1a), ICP was significantly lower in animals treated with methylprednisolone (MTP) than the other groups 30 minutes after induction of SAH. Although a strong trend is visible that animals of the minocycline (MC) group tended to higher ICP values from $60-180$ minutes after induction of $\mathrm{SAH}$, the differences were not significant (1b). Consequently, CPP tended to be lower in the MC group closely missing the level of significance $(p=0.06$ at 30 and 60 minutes after SAH). The values depicted are mean \pm SD. $\left({ }^{*} p<0.05\right)$ 
In the control group, ICP increased to a maximum of $27 \pm 17 \mathrm{mmHg} 15$ minutes after induction of $\mathrm{SAH}$ and gradually declined to $12 \pm 11 \mathrm{mmHg}$ at the end of the monitoring period. In the MTP group, ICP increased to a maximum of $30 \pm 17 \mathrm{mmHg}$ immediately after $\mathrm{SAH}$ and decreased to $15 \pm 8 \mathrm{mmHg}$ at the end of the monitoring period.. In the MC group, ICP reached a maximum of $29 \pm 8$ $\mathrm{mmHg}$ after 15 minutes and returned to $20 \pm 14$

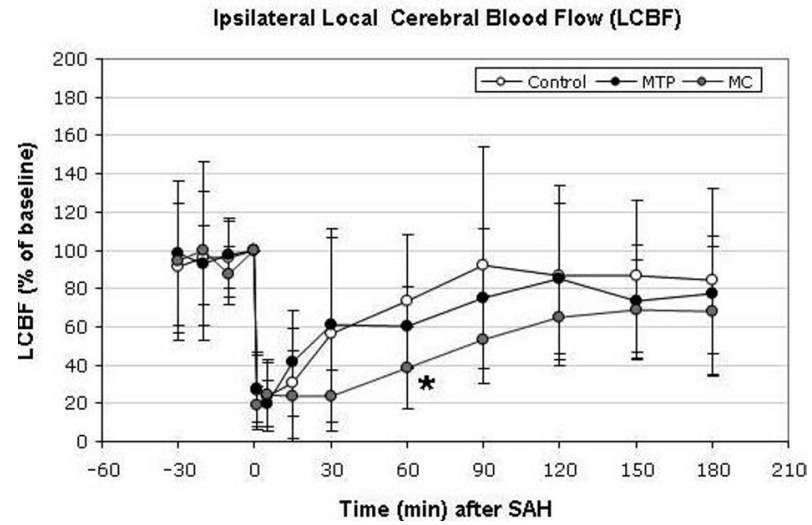

Contralateral Local Cerebral Blood Flow (LCBF)

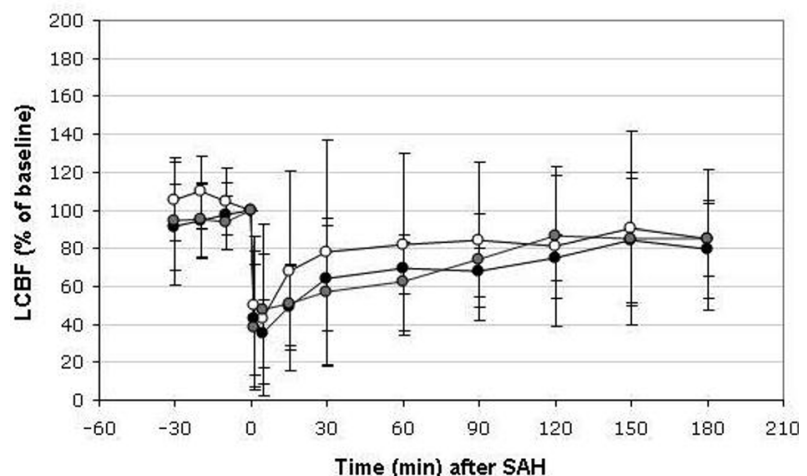

Figure $2 \mathrm{a}$ and b: Course of local cerebral blood flow (LCBF) over the right (ipsilateral) and left (contralateral) hemisphere continuously measured by laser-Doppler flowmetry (LDF) from 30 minutes before until 180 minutes after induction of subarachnoid hemorrhage $(\mathrm{SAH})$. While values in the minocycline $(\mathrm{MC})$ group were lower over the ipsilateral hemisphere reaching the level of significance 60 minutes after endovascular vessel perforation (2a) there was no marked difference over the contralateral hemisphere ( $2 b)$. The values are relative to baseline and depicted as mean \pm SD (*p $<0.05)$.

Table 1. Arterial blood gases before and in hourly intervals after induction of SAH. Differences were not significant throughout the monitoring time.

\begin{tabular}{cccccc}
\hline & & MTP & MC & Control & p \\
\hline Before & $\mathrm{pH}$ & $7.37 \pm 0.05$ & $7.40 \pm 0.05$ & $7.39 \pm 0.06$ & 0.671 \\
& $\mathrm{pO}_{2}$ & $144.4 \pm 24.5$ & $132.5 \pm 20.1$ & $139.0 \pm 21.0$ & 0.734 \\
& $\mathrm{pCO}_{2}$ & $39.7 \pm 4.6$ & $36.3 \pm 8.8$ & $44.5 \pm 5.9$ & 0.118 \\
60 min. & $\mathrm{pH}$ & $7.33 \pm 0.05$ & $7.36 \pm 0.06$ & $7.38 \pm 0.06$ & 0.319 \\
& $\mathrm{pO}_{2}$ & $144.4 \pm 24.5$ & $132.5 \pm 20.1$ & $139.0 \pm 21.0$ & 0.734 \\
& $\mathrm{pCO}_{2}$ & $42.7 \pm 5.6$ & $40.1 \pm 5.3$ & $43.1 \pm 8.7$ & 0.719 \\
& $\mathrm{pH}^{20} \mathrm{~min}$. & $7.38 \pm 0.04$ & $7.36 \pm 0.05$ & $7.38 \pm 0.05$ & 0.543 \\
& $\mathrm{pO}_{2}$ & $115.6 \pm 24.9$ & $108.8 \pm 21.4$ & $116.0 \pm 18.2$ & 0.904 \\
& $\mathrm{pCO}_{2}$ & $40.7 \pm 5.6$ & $41.4 \pm 3.2$ & $43.1 \pm 6.3$ & 0.459 \\
\hline
\end{tabular}

$\mathrm{mmHg} 180$ minutes after the induction of $\mathrm{SAH}$. Differences were not significant at any time point throughout the experiment (Figure 1b).

Baseline values of CPP were $65 \pm 27,60 \pm 11$ and $60 \pm 20 \mathrm{mmHg}$ in the control group, MTP group and MC group, respectively. CPP reached a minimum of $49 \pm 26 \mathrm{mmHg}$ in the control group immediately after $\mathrm{SAH}$ and recovered to baseline values after 60 minutes to reach a final value of $67 \pm 23 \mathrm{mmHg}$ after 180 minutes. In the MTP group, CPP decreased to $36 \pm 26$ $\mathrm{mmHg}$ immediately after $\mathrm{SAH}$ and recovered to $56 \pm 29 \mathrm{mmHg}$ after 3 hours of monitoring. CPP decreased to a minimum of $41 \pm 18 \mathrm{mmHg}$ after the induction of SAH and recovered to $47 \pm 33$ $\mathrm{mmHg}$ at the end of the monitoring period. Differences just barely missed the level of significance 30 and 60 minutes after $\mathrm{SAH}$ with a markedly lower CPP in the MC group compared to the control and MTP groups (Figure 1c).

\section{Local cerebral blood flow}

In control animals, the ipsilateral LCBF declined to $27 \pm 20 \%$ of baseline immediately after vessel perforation. Over the following 90 minutes, it gradually recovered to reach values between 80 and $90 \%$ of baseline thereafter ( 84 $\pm 48 \%$ of baseline at the end of the monitoring time). In the MTP group, LCBF dropped to a mean of $28 \pm 18 \%$ of baseline immediately after SAH and gradually recovered to $77 \pm 31 \%$ of baseline after 180 minutes. In the MC group, LCBF decreased to $18 \pm 10 \%$ of baseline after induction of SAH and slowly recovered to $68 \pm$ $34 \%$ of baseline after 180 minutes (Figure $2 a$ ).

In the contralateral hemisphere, LCBF dropped to $49 \pm 36 \%$ of baseline after induction of SAH and recovered to $84 \pm 37 \%$ at the end of the monitoring period. In the MTP group and MC group, LCBF declined to $43 \pm 36 \%$ and $38 \pm$ $33 \%$ of baseline, respectively and recovered to $79 \pm 26 \%$ and $85 \pm 19 \%$ of baseline at the end of the monitoring period, respectively (Figure $2 \mathrm{~b}$ ).

\section{Extent of hemorrhage, mortality neurological performance}

The average extent of SAH was $10.0 \pm 3.03$ in the MTP group and $8.27 \pm 2.83$ in the control group, classified as moderate according to the classification of Sugawara et al. The MC animals showed an average extent of hemorrhage SAH 
of $7.40 \pm 2.69$, resembling mild hemorrhage according to Sugawara's classification (Figure 3). Differences were not significant ( $p=0.16$ ).

Six animals of the control group, three animals of the MTP group and four animals of the MC group did not survive the first 24 hours after $\mathrm{SAH}$, resulting in an overall mortality of $42 \%$. Differences between the groups were not statistically significant. None of the surviving animals showed signs of hemiparesis as assessed by limb movement and forepaw stretching. Results of activity assessment 24 hours after SAH are depicted in Figure 4. Differences between the groups regarding the activity score did not reach the level of significance $(p=0.42)$.

\section{Histological damage}

The percentage of Caspase 3 positive cells in the hippocampal CA1 region was analyzed in animals that survived 24 hours after $\mathrm{SAH}(\mathrm{n}=5$ in the control group, $n=7$ in the MTP group and $n$ $=6$ in the MC group). Hippocampal damage was significantly reduced in the MTP and MC group compared to the control group (Figure 5).

\section{Discussion}

In these experiments, a well established and standardized model of experimental SAH in rats was used to assess the neuroprotective potential of MTP and MC, two drugs that have long been approved by pharmacological agencies, are readily available and have a well known safety profile. The results suggest that early treatment with anti-inflammatory drugs can reduce early brain damage and improve mortality and neurological performance after experimental SAH.

The endovascular filament model is particularly useful to assess early pathophysiological changes and to compare them with parameters of early brain damage [22]. It was the particular aim of this work in the framework of a larger project to investigate whether there is a beneficial effect of treatment in the early phase of the disease. The substantial disadvantage of the endovascular perforation model is its high mortality as confirmed in the present experiments [17, 23]. However, compared to other studies using this

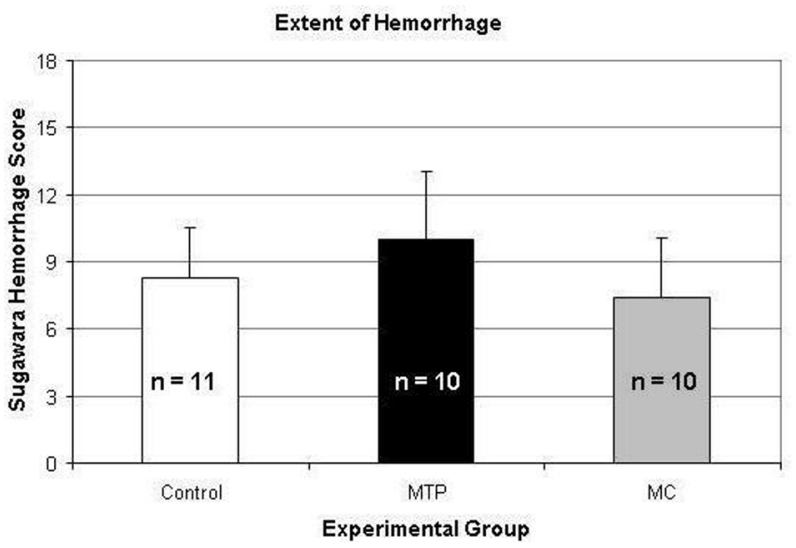

Figure 3: Assessment of the amount of blood in the subarachnoid space using a semiquantitative scale introduced by Sugawara et al. Differences were not statistically significant

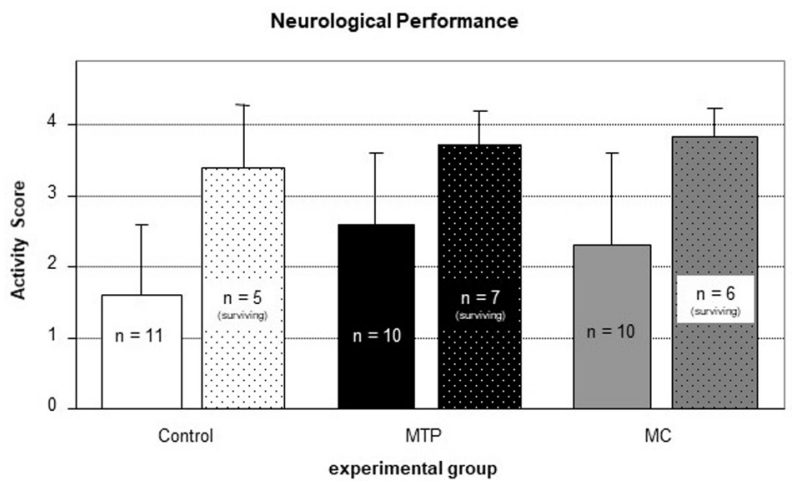

Figure 4: Neurological assessment 24 hours after induction of $\mathrm{SAH}$ by a 5-grade activity score including animals that died before the scheduled timepoint of neurological assessment $(0=$ dead, $4=$ normal activity). These animals as zero points (Solid bars). Scattered bars indicate the neurological performance of the animals that survived the first 24 hours after SAH. Depicted are mean values \pm SD.. Differences did not reach the level of significance.

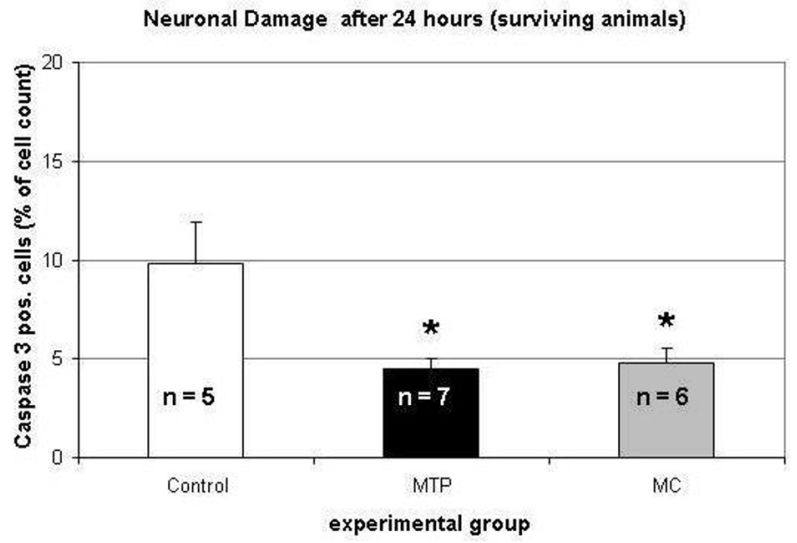

Figure 5: Analysis of hippocampal (CA1) damage by immunohistochemical Caspase 3 staining. Values represent the rate of caspase 3 positive cells of the total cell count per visual field under 40 -fold magnification $\left({ }^{*} p<0.05\right)$.

same model, pathophysiological changes were relatively mild in this series of animals [24]. In particular, ICP increased only moderately, the reason for which may be a relatively low arterial blood pressure $(70-80 \mathrm{mmHg})$ prior to the induction of SAH. Lower baseline blood pressure may result in less extravasation in the case of vessel perforation. This may explain 
the relatively low extent of extravasated blood in the subarachnoid space which was semiquantitatively assessed by the scoring system suggested by Sugawara et al. [20].

Although brain edema is predominantly cytotoxic in the acute stage of $\mathrm{SAH}$, the early reduction of ICP in MTP-treated animals may be a short-term antiedematous effect of the corticosteroid, possibly by the inhibition of natriuresis [25-27]. Higher ICP and lower CPP and ipsilateral LCBF in the group treated with $M C$ can hardly be explained by the pharmacological profile of the drug but rather with a limited number of 2 animals showing a particular increase of ICP after the induction of SAH. In the present sample sizes, this may result in a marked difference, however not statistically significant.

The observation that not only MTP but also MC offered a beneficial effect, although LCBF was reduced in the latter, underlines that immunological mechanisms may strongly contribute to early brain damage after SAH and their treatment in an early stage and in adequate dosage may exert a protective effect.

The pathophysiological cascade of early brain injury after SAH is complex offering a variety of possible targets for therapeutical intervention $[28,29]$. Extravasated hemoglobin, damaged endothelial barrier and free oxygen radicals lead to the formation of brain edema and activation of an inflammatory response that ultimately enhance or result in an uncontrolled apoptosis and/or necrosis [30, 31]. In the last decade, the knowledge about the pathomechanisms of EBI has increased, but no early neuroprotective treatment after $\mathrm{SAH}$ has been introduced into clinical practice. In the present study, we investigated two anti-inflammatory drugs that have been in clinical use for many decades and are, thus ready for use.

MTP has been found to inhibit the induction of apoptosis, represses pro-inflammatory cells and mediators and the induction of suppressor cells [32]. In addition, it attenuates lipid peroxidation, reduces oxidative stress and stabilizes the endothelial barrier [33].

MTP has been used previously in the management of SAH in order to prevent or treat delayed cerebral vasospasm [34-37]. In a metaanalysis of 2005 , though, Feigin et al. concluded that the evidence was not sufficient to support its routine use for prophylaxis of delayed cerebral ischema [38]. In 2010, Gomis et al. published the results of a randomized controlled trial in which they found that early high-dose treatment with MTP resulted in a significantly improved long-term outcome after aneurysmal SAH [12]. In analogy, Czorlich et al. recently reported that treatment with Dexamethasone resulted in a significant improved neurological recovery 4 months after aneurysmal SAH [39].

Similarly, $M C$ has a wide range of antiinflammatory and potentially neuroprotective qualities. In experimental models of various neurologic diseases, positive effects upon on apoptotic, inflammatory and oxidative signaling pathways have been observed [4042]. These may be the result of its ability to down-regulate matrix metalloproteinase (MMP) 9, phospholipase A2 and interleukin (IL-) $1 \beta$ converting enzyme, which play a major role in inflammatory and membrane destabilizing pathways [16, 43-45]. Furthermore, the direct inhibition of cytokines like tumor necrosis factor $a$, interleukin 6 or interleukin $1 \beta$ and the attenuation of caspase dependent and independent apoptosis are further effects of the tetracycline derivate $M C[13,14,35,46]$. In addition, $\mathrm{MC}$ is a potent iron chelator forming iron complexes with the potential to attenuate the inflammatory response by the reduction of its neurotoxic stimulus [14].

Theoretically, beneficial effects of MTP could also be based on a CBF enhancing capacity via inhibition of prostaglandin synthesis [10], and leukocyte-endothelium interactions [11]. Similarly, MC may stabilize CBF via inhibition of MMP-9, global microglia activation, and adhesion/diapedesis of leukocytes $[45,47,48]$.

In the present study, an improvement of CBF was not observed in the treatment groups. In contrast, the treatment groups showed a tendency to lower CBF levels, suggesting that the improvement of neurological performance and attenuation of hippocampal damage are the result of intrinsic anti-inflammatory actions of those two drugs.

$\mathrm{EBI}$ after SAH seems to play a major role in the development of bad outcome in this patient population $[49,50]$. In a number of studies it has been shown that inflammatory pathways, oxidative damage caused by free oxygen radicals and disruption of the blood brain barrier lead to uncontrolled apoptosis and necrosis and, therefore, may result in neuronal damage $[51,52]$. The therapy with MTP reduced the amount of caspase 3 positive cells, a marker of apoptotic transformation, in the animals' hippocampus. The inhibition of various mechanisms modulating inflammatory reactions like transcription factor NF kappa B [10] and lipid peroxidation are possible mechanisms responsible for this finding $[33,53]$.

MC also reduces hippocampal damage 24 hours after $\mathrm{SAH}$, as well. The neuroprotective potential of the tetracycline antibiotic has been reported for a number of different neurological disorders [54]. The results of this study are in accordance with previous results obtained in experimental studies of SAH. Using a double injection model in rats, Guo and coworkers concluded that MC may also reduce EBI by inhibiting MMP-9 [43]. Furthermore, it may, in higher doses, reduce brain edema after $\mathrm{SAH}$ induced by the endovascular perforation model in rats [55]. Recently, Li et al. published data that showed a reduction of apoptotic markers such as p53 and Bax as well as inflammatory target proteins like IL-1 $\beta$ and NLRP3 inflammasome [56].

This study has clear limitations with respect to the small sample size and the early assessment of endpoints. As stated above this series is part of a large project assessing the positive effects of a variety of drugs and mechanisms which are potentially neuroprotective considering the current knowledge about the pathophysiology of EBI. To elaborate differences on physiological parameters including CBF these sample sizes are usually adequate. These parameters were not influenced by therapy with MTP and MC, respectively. To show an improvement of neurological performance, sample sizes are too small. The main finding is the improvement of hippocampal damage after 24 hours in spite of a trend to lower CBF values. This timepoint may be relatively early but it was the distinct aim of this project to address the effects of early brain damage after SAH and not mix up with any form of delayed ischemic process. Pathophysiological changes in rodent models may proceed more rapidly than in humans. 
Therefore, the endpoint for neurological assessment and histological assessment was deliberately chosen.

\section{Conclusion}

In this experimental study, an attenuation of neuronal damage has been observed by therapy with the corticosteroid MTP and the tetracycline MC. Both substances have a number of potentially neuroprotective qualities. Since physiological parameters were not improved by treatment with either substance, we conclude that the strong antiinflammatory action is most likely to be the key factor for the beneficial effects.

\section{Acknowledgements}

This publication was funded by the German Research Foundation (DFG) and the University of Wuerzburg in the funding programme Open Access Publishing.

\section{References}

[1] Macdonald RL, Higashida RT, Keller E, Mayer SA, Molyneux A, Raabe A et al., Clazosentan, an endothelin receptor antagonist, in patients with aneurysmal subarachnoid haemorrhage undergoing surgical clipping: a randomised, double-blind, placebo-controlled phase 3 trial (CONSCIOUS-2), Lancet Neurol 2011, 10, 618-625.

[2] Sehba FA, Pluta RM, Zhang JH, Metamorphosis of subarachnoid hemorrhage research: from delayed vasospasm to early brain injury, Mol Neurobiol 2011, 43, 27-40.

[3] van Donkelaar CE, Bakker NA, Veeger NJ, Uyttenboogaart $M$, Metzemaekers JD, Eshghi $O$ et al., Prediction of outcome after subarachnoid hemorrhage: timing of clinical assessment, J Neurosurg 2017, 126, 52-59.

[4] Suwatcharangkoon S, Meyers E, Falo C, Schmidt JM, Agarwal S, Claassen $J$ et al., Loss of Consciousness at Onset of Subarachnoid Hemorrhage as an Important Marker of Early Brain Injury, JAMA Neurol 2016, 73, 28-35.

[5] Schubert GA, Schilling L, Thome C, Clazosentan, an endothelin receptor antagonist, prevents early hypoperfusion during the acute phase of massive experimental subarachnoid hemorrhage: a laser Doppler flowmetry study in rats, J Neurosurg 2008, 109, 1134-1140.

[6] Seifert V, Loffler BM, Zimmermann M, Roux S, Stolke D, Endothelin concentrations in patients with aneurysmal subarachnoid hemorrhage. Correlation with cerebral vasospasm, delayed ischemic neurological deficits, and volume of hematoma, J Neurosurg 1995, $82,55-62$.

[7] Sercombe R, Dinh YR, Gomis P, Cerebrovascular inflammation following subarachnoid hemorrhage, Jpn J Pharmacol 2002, 88, 227249.

[8] Cahill J, Calvert JW, Zhang JH, Mechanisms of early brain injury after subarachnoid hemorrhage, J Cereb Blood Flow Metab 2006, 26, 1341-1353.

[9] Hasegawa Y, Suzuki H, Sozen T, Altay O, Zhang JH, Apoptotic mechanisms for neuronal cells in early brain injury after subarachnoid hemorrhage, Acta Neurochir Suppl 2011, 110, 43-48.

[10] Almawi WY, Melemedjian OK, Negative regulation of nuclear factorkappaB activation and function by glucocorticoids, J Mol Endocrinol 2002, 28, 69-78.

[11] Yamakawa K, Sasaki T, Tsubaki S, Nakagomi T, Saito I, Takakura K, Effect of high-dose methylprednisolone on vasospasm after subarachnoid hemorrhage, Neurol Med Chir (Tokyo) 1991, 31, 24-31.

[12] Gomis P, Graftieaux JP, Sercombe R, Hettler D, Scherpereel B,
Rousseaux P, Randomized, double-blind, placebo-controlled, pilot trial of high-dose methylprednisolone in aneurysmal subarachnoid hemorrhage, J Neurosurg 2010, 112, 681-688.

[13] Elewa HF, Hilali H, Hess DC, Machado LS, Fagan SC, Minocycline for short-term neuroprotection, Pharmacotherapy 2006, 26, 515-521.

[14] Bahrami F, Morris DL, Pourgholami MH, Tetracyclines: drugs with huge therapeutic potential, Mini Rev Med Chem 2012, 12, 44-52.

[15] Saivin S, Houin G, Clinical pharmacokinetics of doxycycline and minocycline, Clin Pharmacokinet 1988, 15, 355-366.

[16] Stirling DP, Koochesfahani KM, Steeves JD, Tetzlaff W, Minocycline as a neuroprotective agent, Neuroscientist 2005, 11, 308-322.

[17] Bederson JB, Germano IM, Guarino L, Cortical blood flow and cerebral perfusion pressure in a new noncraniotomy model of subarachnoid hemorrhage in the rat, Stroke 1995, 26, 1086-1091.

[18] Lilla N, Hartmann J, Koehler S, Ernestus RI, Westermaier T, Early NOdonor treatment improves acute perfusion deficit and brain damage after experimental subarachnoid hemorrhage in rats, J Neurol Sci 2016, 370, 312-319.

[19] Garcia JH, Wagner S, Liu KF, Hu XJ, Neurological deficit and extent of neuronal necrosis attributable to middle cerebral artery occlusion in rats. Statistical validation, Stroke 1995, 26, 627-634.

[20] Sugawara T, Ayer R, Jadhav V, Zhang JH, A new grading system evaluating bleeding scale in filament perforation subarachnoid hemorrhage rat model, J Neurosci Methods 2008, 167, 327-334.

[21] Paxinos G, Watson C, The Rat Brain in stereotaxic Coordinates, 2005.

[22] Prunell GF, Mathiesen T, Diemer NH, Svendgaard NA, Experimental subarachnoid hemorrhage: subarachnoid blood volume, mortality rate, neuronal death, cerebral blood flow, and perfusion pressure in three different rat models, Neurosurgery 2003, 52, 165-175.

[23] Veelken JA, Laing RJ, Jakubowski J, The Sheffield model of subarachnoid hemorrhage in rats, Stroke 1995, 26, 1279-1283.

[24] Westermaier T, Jauss A, Eriskat J, Kunze E, Roosen K, Acute vasoconstriction: decrease and recovery of cerebral blood flow after various intensities of experimental subarachnoid hemorrhage in rats, J Neurosurg 2009, 110, 996-1002.

[25] Mistry AM, Mistry EA, Ganesh KN, Froehler MT, Fusco MR, Chitale RV, Corticosteroids in the Management of Hyponatremia, Hypovolemia, and Vasospasm in Subarachnoid Hemorrhage: A Meta-Analysis, Cerebrovasc Dis 2016, 42, 263-271.

[26] Nakagawa I, Hironaka Y, Nishimura F, Takeshima Y, Matsuda R, Yamada $S$ et al., Early inhibition of natriuresis suppresses symptomatic 
cerebral vasospasm in patients with aneurysmal subarachnoid hemorrhage, Cerebrovasc Dis 2013, 35, 131-137.

[27] Moro N, Katayama Y, Kojima J, Mori T, Kawamata T, Prophylactic management of excessive natriuresis with hydrocortisone for efficient hypervolemic therapy after subarachnoid hemorrhage, Stroke 2003, 34, 2807-2811.

[28] Sehba FA, Bederson JB, Mechanisms of acute brain injury after subarachnoid hemorrhage, Neurol Res 2006, 28, 381-398.

[29] Sehba FA, Mostafa G, Knopman J, Friedrich VJ, Bederson JB, Acute alterations in microvascular basal lamina after subarachnoid hemorrhage, J Neurosurg 2004, 101, 633-640.

[30] Chaichana KL, Pradilla G, Huang J, Tamargo RJ, Role of inflammation (leukocyte-endothelial cell interactions) in vasospasm after subarachnoid hemorrhage, World Neurosurg 2010, 73, 22-41.

[31] Sabri M, Ai J,LakovicK, Macdonald RL, Mechanisms of microthrombosis and microcirculatory constriction after experimental subarachnoid hemorrhage, Acta Neurochir Suppl 2013, 115, 185-192.

[32] Schweingruber N, Reichardt SD, Luhder F, Reichardt HM, Mechanisms of glucocorticoids in the control of neuroinflammation, J Neuroendocrinol 2012, 24, 174-182.

[33] Bracken MB, Shepard MJ, Collins WF, Holford TR, Young W, Baskin DS et al., A randomized, controlled trial of methylprednisolone or naloxone in the treatment of acute spinal-cord injury. Results of the Second National Acute Spinal Cord Injury Study, N Engl J Med 1990, 322, 1405-1411.

[34] Fox JL, Yasargil MG, The relief of intracranial vasospasm: an experimental study with methylprednisolone and cortisol, Surg Neurol 1975, 3, 214-218.

[35] Chen D, Nishizawa S, Yokota N, Ohta S, Yokoyama T, Namba H, Highdose methylprednisolone prevents vasospasm after subarachnoid hemorrhage through inhibition of protein kinase $\mathrm{C}$ activation, Neurol Res 2002, 24, 215-222.

[36] Chyatte D, Fode NC, Nichols DA, Sundt TMJ, Preliminary report: effects of high dose methylprednisolone on delayed cerebral ischemia in patients at high risk for vasospasm after aneurysmal subarachnoid hemorrhage, Neurosurgery 1987, 21, 157-160.

[37] Chyatte D, Rusch N, Sundt TMJ, Prevention of chronic experimental cerebral vasospasm with ibuprofen and high-dose methylprednisolone, J Neurosurg 1983, 59, 925-932.

[38] Feigin VL, Anderson N, Rinkel GJ, Algra A, van Gijn J, Bennett DA, Corticosteroids for aneurysmal subarachnoid haemorrhage and primary intracerebral haemorrhage, Cochrane Database Syst Rev 2005, CD004583.

[39] Czorlich P, Sauvigny T, Ricklefs F, Abboud T, Nierhaus A, Vettorazzi $E$ et al., Impact of dexamethasone in patients with aneurysmal subarachnoid haemorrhage, Eur J Neurol 2017, 24, 645-651.

[40] Garrido-Mesa N, Zarzuelo A, Galvez J, Minocycline: far beyond an antibiotic, Br J Pharmacol 2013, 169, 337-352.

[41] Mori K, Maeda M, Miyazaki M, Iwase $H$, Misery perfusion caused by cerebral hypothermia improved by vasopressor administration, Neurol Res 1999, 21, 585-592.
[42] Morimoto $N$, Shimazawa M, Yamashima T, Nagai $H$, Hara $H$, Minocycline inhibits oxidative stress and decreases in vitro and in vivo ischemic neuronal damage, Brain Res 2005, 1044, 8-15.

[43] Guo ZD, Wu HT, Sun XC, Zhang XD, Zhang JH, Protection of minocycline on early brain injury after subarachnoid hemorrhage in rats, Acta Neurochir Suppl 2011, 110, 71-74.

[44] Dalm D, Palm GJ, Aleksandrov A, Simonson T, Hinrichs W, Nonantibiotic properties of tetracyclines: structural basis for inhibition of secretory phospholipase A2, J Mol Biol 2010, 398, 83-96.

[45] Koistinaho M, Malm TM, Kettunen Ml, Goldsteins G, Starckx S, Kauppinen RA et al., Minocycline protects against permanent cerebral ischemia in wild type but not in matrix metalloprotease-9deficient mice, J Cereb Blood Flow Metab 2005, 25, 460-467.

[46] Heo K, Cho YJ, Cho KJ, Kim HW, Kim HJ, Shin HY et al., Minocycline inhibits caspase-dependent and -independent cell death pathways and is neuroprotective against hippocampal damage after treatment with kainic acid in mice, Neurosci Lett 2006, 398, 195-200.

[47] Tikka T, Fiebich BL, Goldsteins G, Keinanen R, Koistinaho J, Minocycline, a tetracycline derivative, is neuroprotective against excitotoxicity by inhibiting activation and proliferation of microglia, J Neurosci 2001, 21, 2580-2588.

[48] Brundula V, Rewcastle NB, Metz LM, Bernard CC, Yong VW, Targeting leukocyte MMPs and transmigration: minocycline as a potential therapy for multiple sclerosis, Brain 2002, 125, 1297-1308.

[49] Sehba FA, Hou J, Pluta RM, Zhang JH, The importance of early brain injury after subarachnoid hemorrhage, Prog. Neurobiol 2012, 97, 1437.

[50] Caner B, Hou J, Altay O, Fujii M, Zhang JH, Transition of research focus from vasospasm to early brain injury after subarachnoid hemorrhage, J Neurochem 2012, 123 Suppl, 12-21.

[51] Ostrowski RP, Colohan AR, Zhang JH, Molecular mechanisms of early brain injury after subarachnoid hemorrhage, Neurol Res 2006, 28, 399-414.

[52] Miller BA, Turan N, Chau M, Pradilla G, Inflammation, vasospasm, and brain injury after subarachnoid hemorrhage, Biomed Res Int 2014, 2014, 384342.

[53] Lombardi D, Gaetani P, Marzatico F, Cafe C, Rodriguez, Effect of high-dose methylprednisolone on anti-oxidant enzymes after experimental SAH, J Neurol Sci 1992, 111, 13-19.

[54] Matsukawa N, Yasuhara T, Hara K, Xu L, Maki M, Yu G et al., Therapeutic targets and limits of minocycline neuroprotection in experimental ischemic stroke, BMC Neurosci 2009, 10:126, -10.

[55] Sherchan P, Lekic T, Suzuki H, Hasegawa Y, Rolland W, Duris K et al., Minocycline improves functional outcomes, memory deficits, and histopathology after endovascular perforation-induced subarachnoid hemorrhage in rats, J Neurotrauma 2011, 28, 25032512.

[56] Li J, Chen J, Mo H, Qian C, Yan F, Gu C et al., Minocycline Protects Against NLRP3 Inflammasome-Induced Inflammation and P53Associated Apoptosis in Early Brain Injury After Subarachnoid Hemorrhage, Mol Neurobiol 2016, 53, 2668-2678. 